\section{Animais Solidários: A Zooterapia como Extensão Universitária para Idosos Institucionalizados}

\author{
Solidary Animals: The Zootherapy as University Extension \\ to Institutionalized Elders
}

\section{RESUMO}

O objetivo deste estudo foi introduzir diferentes animais no cotidiano de idosos do asilo São Vicente de Paulo, na cidade de Pirassununga, estado de São Paulo, através do projeto Animais Solidários da Faculdade de Medicina Veterinária e Zootecnia da Universidade de São Paulo, buscando identificar uma forma de melhorar a sociabilização, humor, cognição e bem-estar dos idosos institucionalizados. $\mathrm{O}$ envelhecimento populacional representa um grande desafio para os familiares, gestores de saúde e instituições que abrigam os idosos. Associado ao processo de envelhecimento, o declínio da capacidade física e cognitiva, a depender de sua extensão, pode levar o idoso a perda da independência, da autonomia e a uma profunda tristeza. Esse quadro gera, para muitos idosos e familiares, a necessidade de institucionalização. A internação, por sua vez, pode levar ao agravamento dessas condições, quando sob determinadas circunstâncias. Foi realizado estudo descritivo, exploratório e transversal, abrangendo trinta e quatro idosos de idade média de setenta e seis anos, de ambos os sexos. Foi evidenciado que $82 \%$ dos idosos já tinham tido experiência e convívio com os animais, 63\% tinham vontade de possuir um animal, $84 \%$ dos funcionários foram favoráveis à visitação dos mesmos; porém, $81 \%$ não foram favoráveis à permanência dos mesmos na instituição. Encontrou-se também que 82,35\% dos idosos (ou seja, vinte e oito) participaram de todas as atividades propostas, sendo constatadas melhoras quanto à socialização e aumento do afeto para com os animais e a equipe executora do projeto. $\mathrm{O}$ avanço dos estudos na área de Zooterapia e intervenções da interação homem-animal com idosos institucionalizados é um passo importante para tal campo do conhecimento, assim como uma maior atenção à leitura e interpretação das respostas dos idosos, uma vez que alguns deles apresentam déficits cognitivos.

Palavras-chave: Zooterapia. Idoso Institucionalizado. Interações. Bem-estar Animal.

\section{Gisele Dela Ricci, Carina TORELli, Maria de Fátima Martins e Thiago William de Almeida \\ Universidade de São Paulo. Faculdade de Medicina Vete- rinária e Zootecnia, São Paulo, Brasil}




\section{ABSTRACT}

This study aimed to introduce different animals into Vicente de Paulo Asylum quotidian, in Pirassununga city, State of São Paulo, through the lonely animals' project of veterinary medicine and husbandry faculty of São Paulo State University, researching identify the best way to improve socialization, cognition and well-being of elders. The aging of the population represent a great challenge to relatives, health managers and institutions that harbor the elders. Associated to aging process, the decline of physics and cognate ability associated to institutionalization takes the elder to an independent and autonomy loss and sometimes deep sadness. A description, exploratory and transversal, hugging elder of seventy six years-old average of both sex. It was used the Qui-square test $\left(\mathrm{x}^{2}\right)$ to statistics analysis of related factors to empathy with animals and preference of species and the $t$ student test that set the significance of different among the groups established in $\mathrm{p}<0,05$ of thirty four elder total that participated of the study, It was showed that $82 \%$ of the elders have already had experience and familiarity with animals, $63 \%$ had desire of having an animal, $84 \%$ of the employees were favorable to the visiting of the animals, but $81 \%$ did not accepted the permanence of the same. Were found as well that $82,35 \%$ participated of all proposed activities and improved the socialization and affection with the animals and the team that developed the project. Suggests others studies in the husbandry field and interventions oh human-animal with institutionalized elders, hence, biggest attentions must be done to reading and comprehension of elders answers, once them, presents cognate deficits.

Keywords: Husbandry. Institutionalized Elders. Interactions. Animal Well-being.

\section{INTRODUÇÃO}

\section{O envelhecimento da população brasileira é uma realidade com a qual nos} deparamos nos dias atuais e representa um desafio para os familiares, gestores de saúde e instituições que abrigam idosos, conhecidas como asilos ou casas de repouso.

Ramos [11] indica que a velhice representa a fase da vida onde há uma alta incidência de doenças crônicas não transmissíveis, limitações físicas e perdas refletidas em isolamento social, declínio sensorial e cognitivo.

A evolução e as complicações do envelhecimento variam de acordo com a história de vida de cada idoso e se processam dentro do seu contato familiar e social, não dependendo somente, então, de aspectos biológicos, mas também de fatores como os ambientais, econômicos, culturais e sociais.

Frequentemente, a institucionalização de um idoso tem como primeira consequência o despertar de um sentimento de perda da autonomia, onde a liberdade pessoal, independência física e o controle das próprias decisões já não fazem parte da vida dos idosos, tornando-os dependentes de terceiros, ou das normas das instituições acolhedoras [2]. Essas características podem acarretar ao idoso o aumento da susceptibilidade a doenças e à tristeza profunda, respostas biológicas de múltiplas origens: física, psíquica ou social.

Algumas instituições, quando não regidas por princípios de humanização, dão ao 
idoso a consciência do abandono acarretando sofrimento, o que gera sentimento de insegurança, medo, conflitos psicológicos e, também, a perda da vontade de viver.

O contato com um animal pode ser capaz de desencadear um relacionamento livre de estresse, com menor grau de julgamento e de expectativas. A troca de afeições entre humanos e animais não são sentimentos ambivalentes, diferenciando-se das relações entre humanos e seus membros da família, que frequentemente trocam sentimentos e estados emocionais, nem sempre positivos.

$\mathrm{O}$ animal aceita amar incondicionalmente, sem exceções ou considerações, sem analisar o estado de saúde, idade ou deficiências [5]; oferta amor, permitindo a sensação de amizade e bem-estar por contato [8].

A Zooterapia ou Terapia Assistida por Animais é uma ciência de característica multi, inter e transdisciplinar, na qual os animais são utilizados no alívio do estresse, da depressão, da sensação de abandono ou solidão e sociabilização do ser humano.

Essa ciência tem originado benefícios aos diferentes pacientes com problemas relacionados ao sistema cardíaco e imunológico: alívio do estresse, sendo facilitadora do desenvolvimento das habilidades cognitivas e sócioemocionais, auxiliando no combate à depressão, diminuindo a ansiedade e melhorando a coordenação motora, sendo, por exemplo, uma motivação para caminhar e realizar exercícios da fisioterapia [3].

Além da função de companhia e produção, os animais têm exercido, na medicina veterinária, a função de co-terapeutas e co-educadores dentro de uma expectativa de bem-estar e educação humanitária, através de uma educação holística. Essa ciência ainda está em processo de inserção nos meios acadêmicos, e o tema dos idosos institucionalizados tem sido pouco estudado.

A utilização dos animais como zooterapeutas pode ser compreendida pelo paradigma holístico de cuidados a saúde. Holos em grego significa saúde, integridade e totalidade. Desta forma, a filosofia holística de cuidado da saúde implica em enxergar cada pessoa como um todo integrado, consistindo em corpo, mente e espírito, o que torna o todo algo além do que simplesmente uma soma das partes [9].

O projeto de zooterapia para idosos institucionalizados, da Faculdade de Medicina Veterinária e Zootecnia da Universidade de São Paulo, no campus de Pirassununga, constitui-se como um programa de cultura e extensão, seguido da pesquisa científica. A zooterapia enquanto ciência tem ampliado a visão holística dos futuros profissionais que veem a interação dos idosos com animais como importante catalisadora do bem-estar no cotidiano de idosos institucionalizados.

Diante disso, o presente estudo busca verificar existência de afinidade entre animais e idosos institucionalizados, e vislumbra a Zooterapia como nova ciência que pode vir a contribuir de forma holística para o bem-estar destes idosos.

\section{MATERIAL E MÉTODOS}

As práticas com idosos institucionalizados são desenvolvidas no asilo São Vicente de Paulo, uma realização do projeto Animais Solidários da Faculdade de Medicina Veterinária e Zootecnia, apoiado pela Pró-Reitoria de Cultura e Extensão da Universidade de 
São Paulo (PRCEU-USP), no campus de Pirassununga. O projeto também é contemplado pelas disciplinas de Zooterapia da graduação e pós-graduação da mesma unidade.

Participam do projeto de extensão trinta e quatro idosos institucionalizados, sendo dezoito do sexo feminino e dezesseis do sexo masculino, com idade média de setenta e seis anos. Dentre as atividades desenvolvidas está a realização de festas temáticas, como a de natal, carnaval, páscoa e dia do trabalho, com animais caracterizados de acordo com a data comemorada (Figura 1).

Figura 1 - Animal fantasiado para festa de Natal no asilo São Vicente de Paulo (à esquerda). Idoso interagindo com animal (à direita)
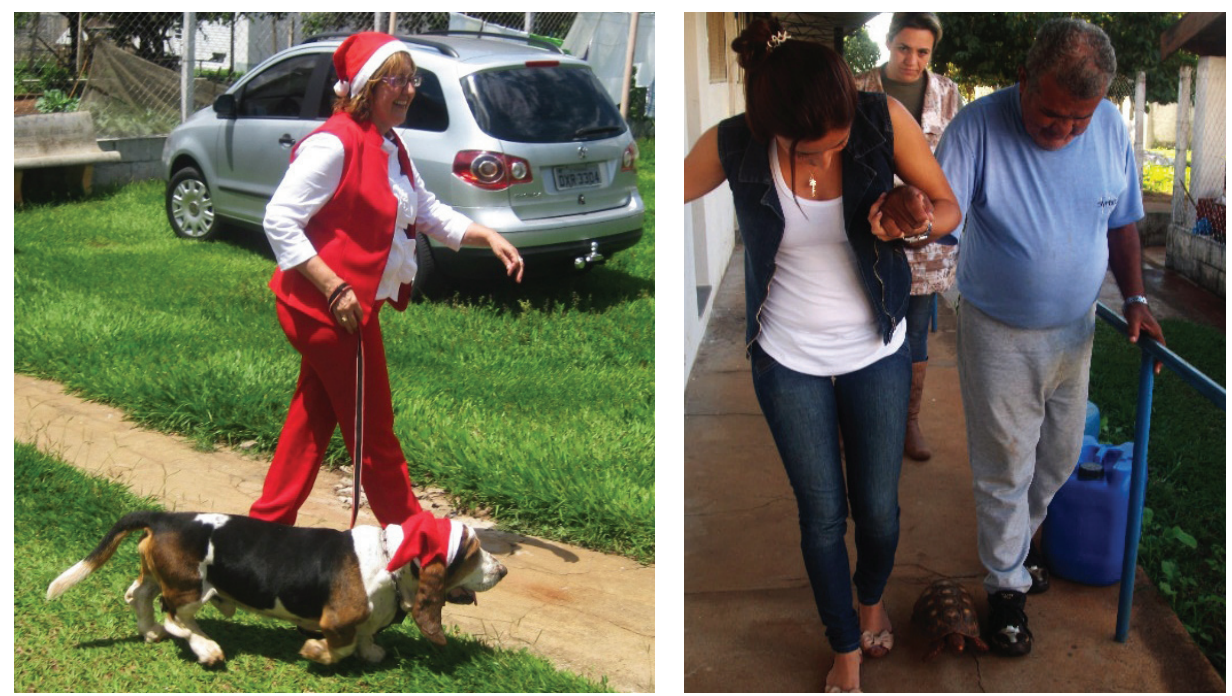

Foi realizada, com essa mesma população, pesquisa do tipo exploratório-descritiva e transversal, com abordagem qualitativa e quantitativa. Esse método foi escolhido por permitir aprofundamento dos objetivos a serem avaliados (idosos institucionalizados e afinidade com os animais) por meio da fala dos entrevistados (questionários qualitativos).

Nas visitas realizadas aos asilos, alguns materiais e dados foram recolhidos para análises estatísticas, como o teste do Qui-Quadrado $\left(\mathrm{X}^{2}\right)$. Para a análise estatística dos fatores relacionados à empatia com os animais e predileção por espécie foram utilizados, também, o Qui-Quadrado e o teste t-Student, que determinou a significância das diferenças entre os grupos estabelecidos em $\mathrm{p}>0,05$.

Foram utilizados e desenvolvidos formulários das atividades a serem desenvolvidas entre idosos e animais, sendo específicos para cada espécie envolvida. Etogramas foram feitos para a avaliação do comportamento e bem-estar dos animais.

Para a autorização de divulgação dos dados obtidos, foi assinado um termo de consentimento da diretoria da instituição para as visitas dos animais, autorização das fotos e a futura exposição dos dados recolhidos. 


\section{Participantes}

O projeto atendeu, entre 2000 e 2013, aproximadamente duzentos e dez alunos de graduação e pós-graduação da disciplina de Zooterapia, sendo desenvolvidos seis trabalhos de conclusão de curso: dois em Zooterapia, um em Psicologia, um em Fisioterapia, dois em Enfermagem e nove em práticas profissionalizantes, sendo dezoito alunos de graduação da Bolsa Trabalho da Superintendência de Assistência Social (SAS-USP), dezenove alunos do programa Aprender com Cultura e Extensão, catorze alunos de pré-iniciação científica, seis alunos de pós-graduação do Programa de Aperfeiçoamento de Ensino (PAE) e cinco visitantes da Universidade de Mossoró, Rio Grande do Norte, além de doze integrantes de diversas ONGs dos estados de São Paulo, Minas Gerais, Rio Grande do Sul e Rio Grande do Norte.

Animais como cães, gatos, peixes, escargots, hamsters, camundongos hair less e pássaros são, também, participantes essenciais para o projeto. Esses animais são treinados para interagir por comando, pegar bolinhas ou brinquedos atirados, andar sobre áreas comuns sem apresentar perigo ou susto, permanecendo calmos e relaxados na presença de muitas pessoas. Os animais realizam visitas quinzenais aos asilos e interagem com os idosos, sendo monitorados pelos alunos e pela supervisora do projeto.

\section{RESULTADOS E DISCUSSÕES}

Do total de idosos assistidos pelo projeto, vinte e quatro tiveram e conviveram com cães antes da institucionalização; onze tiveram gatos; dezenove, pássaros; oito tiveram jabutis; doze, peixes; seis, coelhos; onze possuíam cavalos; quatro, vacas e um possuiu um caracol de jardim. Com isso, pode-se afirmar que $82 \%$ dos idosos já tiveram experiência e convívio com os animais, sendo que $63 \%$ relataram sentir vontade de possuir um animal perto para companhia. Ainda, $84 \%$ das pessoas que trabalham na instituição foram favoráveis à visitação dos animais, porém $81 \%$ não foram favoráveis a sua permanência na instituição.

Observamos que a comunicação entre os idosos institucionalizados aumentou durante a visitação dos animais, sendo que, dos trinta e quatro idosos, vinte e oito interagiram com os animais, incluindo contato físico: receber no colo, afagar, colocar e tirar a coleira, colocar uma gravata ou um laço, escovar, dar um petisco, caminhar com o jabuti, colocar a calopsita no ombro e pintar as conchas dos escargots (Figuras 2 e 3 ). 
Figura 2 - Idoso interagindo com o animal durante a festa de Natal (à esquerda).

À direita, contato carinhoso entre idoso, animal e equipe do projeto.
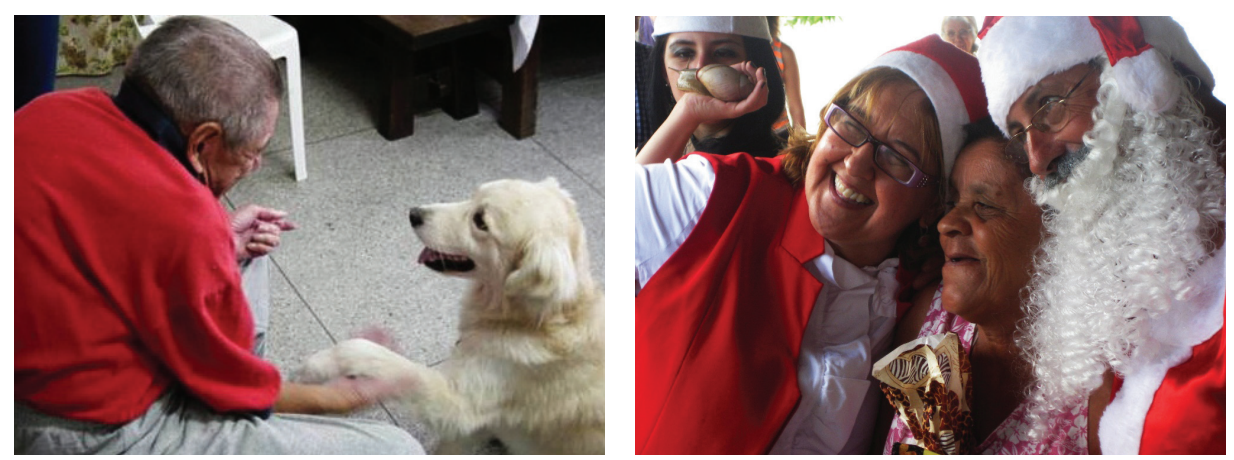

Figura 3 - Relação de afeto entre animais e idosa na instituição (à esquerda).

À direita, atividade lúdica com conchas envolvendo aspectos cognitivos dos idosos.
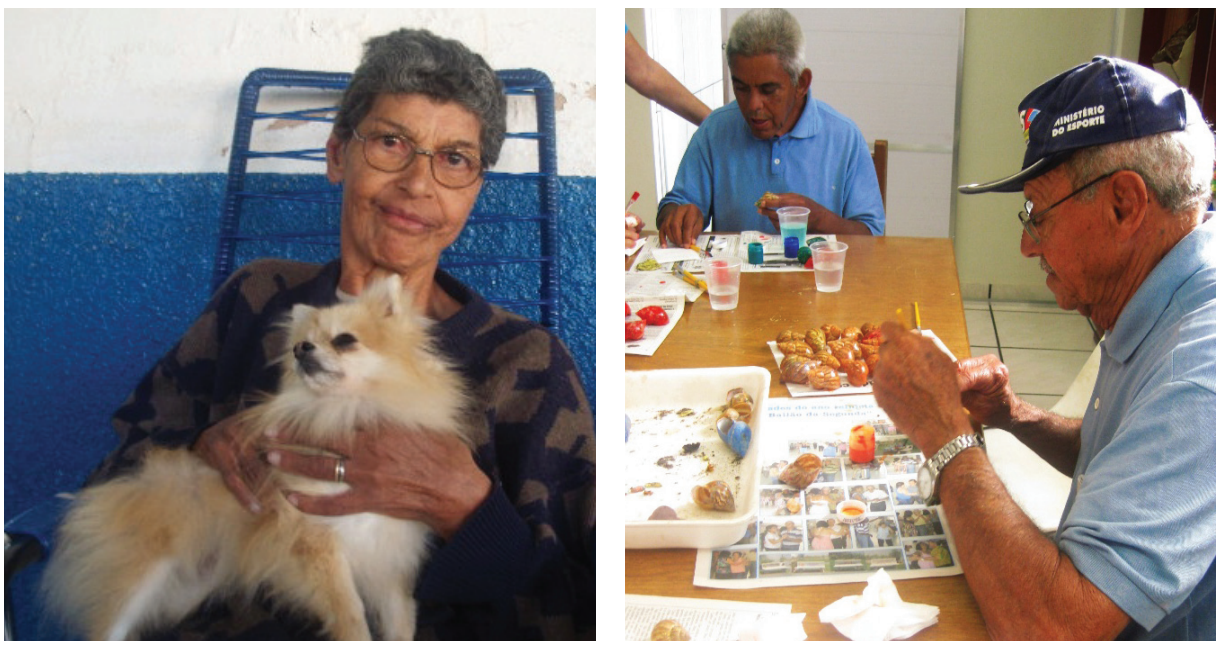

Os benefícios da Zooterapia ou Terapia Assistida por Animais estão pautados na ética solidária e no trabalho de equipes multi, inter e transdisciplinares, interligando aspectos como idade, cultura e crença. Estudos mostram benefícios tangíveis da interação dos animais com grupos de pacientes geriátricos $[6,11]$. No decorrer do desenvolvimento do projeto, foi observada uma comunicação positiva entre os idosos e a equipe do projeto durante a visita dos animais (Figuras 4 e 5 ).

Figura 4 - Contato do idoso institucionalizado, animal e estudantes na casa de repouso (à esquerda).

À direita, atividade física realizada com idoso, jabuti e alunos na instituição.

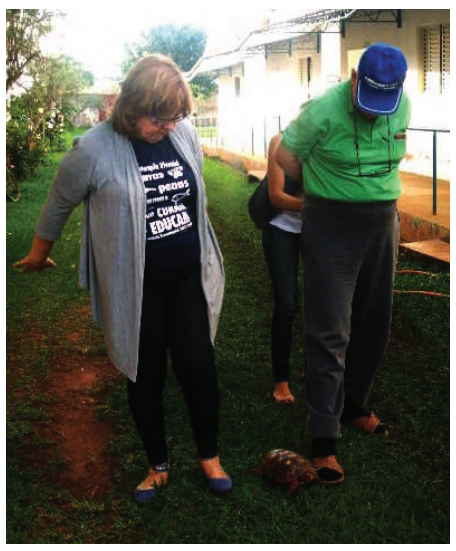

Animais Solidários: A Zooterapia como Extensão Universitária para Idosos Institucionalizados 

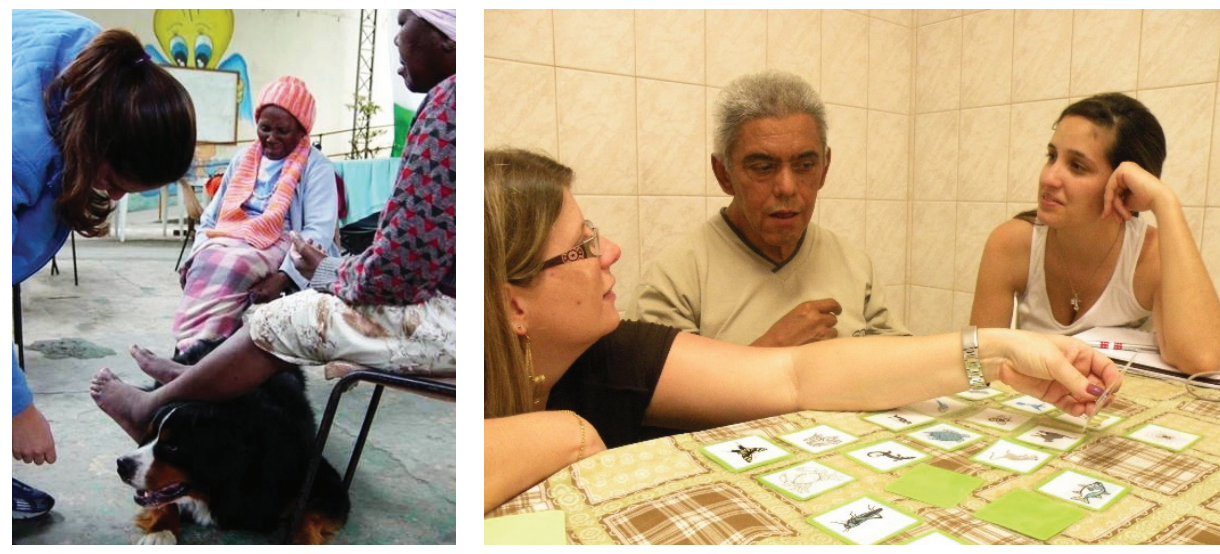

Figura 5 - Atividade física realizada com a coordenadora do projeto e idoso institucionalizado (à esquerda).

À direita, jogo da memória envolvendo alunos e idoso, atividade idealizada pelo projeto.

Uma significante correlação foi observada entre a presença do contato físico e o estabelecimento de relacionamento $(\mathrm{r}=0,45 \mathrm{e} \mathrm{p}=0,06)$. Estes dados se repetiram em proporções coerentes, sendo que a maioria dos idosos (28/34) alcançou relacionamento e proporção positiva nas atividades propostas.

Lermontov [7] constatou em seu estudo que há a redução do uso de medicamentos, menos incidência de depressão e aumento da sobrevivência de enfartos com o uso da Zooterapia. Alguns dos idosos deste projeto relataram que a presença dos animais deixava os seus dias mais alegres. Um deles notificou que não recebia visita dos familiares, porém recebia carinho dos animais, melhorando a sua autoestima e alegria.

Notou-se que nenhum dos idosos que participaram da prática relatou que a atividade foi negativa, sendo que a maioria dos idosos, $81 \%$, considerou a atividade relaxante, tornando-se mais otimista pela presença dos animais. Entre os idosos, 54\% notificaram que tiveram animais no passado, $8 \%$ choraram de saudades dos seus animais e $18 \%$ sentiram que o mais importante benefício foi ter no animal um objeto para a projeção de sentimento.

Todos estes benefícios reportados pelos idosos foram correlacionados com a observação, validando a análise exploratória, descritiva e transversal. O teste t-Student, usado para comparar as médias relacionadas ao desempenho da interação com os animais, mostrou diferenças estatisticamente significativas: $p>0,05$ para os tipos de animais utilizados na pesquisa. Interessante citar que, quanto ao afeto demonstrado para com os animais, o cachorro, o peixe e as calopsitas foram os animais de preferência do sexo masculino e os cães e gatos, do sexo feminino. Uma maior média de sociabilização com a equipe foi evidenciada em relação aos idosos do sexo masculino quando comparados com as idosas do sexo feminino.

Entre os alunos envolvidos, $92 \%$ deles relataram satisfação na participação no projeto e $42 \%$ indicaram que as visitas dos animais têm a função de fazer repensar conceitos sociais como o abandono de familiares.

A metodologia utilizada revelou-se fidedigna para a população avaliada, porém maior atenção deve ser dada a leitura e interpretação das respostas dos idosos, uma vez que alguns deles apresentam déficits cognitivos. 


\section{CONSIDERAÇÕES FINAIS}

O estudo contribuiu para o conhecimento dos efeitos da Zooterapia no cotidiano de idosos institucionalizados. Neste contexto, sugere-se mais estudos a respeito deste tema, assim como o desenvolvimento e implementação de atividades lúdicas e recreativas com animais voltadas aos idosos institucionalizados. Para tanto, o treinamento e aproximação dos alunos universitários devem ser estimulados através de fomentos para que a extensão universitária possa ser usada como uma forma de acrescentar conhecimento e benefício direto e eficaz para a comunidade e, principalmente, esclarecer a importância do vínculo entre o idoso e os animais; temos, finalmente, que o projeto Animais Solidários em instituições de idosos tem sido um celeiro para a aprendizagem e aperfeiçoamento na formação de todos os envolvidos.

\section{REFERÊNCIAS}

[1] ARRUDA, K. P. Beneficios Psicológicos da Equoterapia. Itatiba: USF, 2002.

[2] ARGIMON, I. L., STEN, L. M. Habilidades cognitivas em indivíduos muito idosos: um estudo longitudional. Rio de Janeiro, Cadernos de Saúde Pública, v.21, n.1, p. 64-72, 2005 .

[3] BERGAMO, G. O doutor é animal. Revista Veja on-line, São Paulo, 30 nov. 2005. Disponível em: <http://veja.abril.com.br/301105/p_o66.html> Acesso em: 23 jan. de 2014.

[4] BIGATELlO, G; FRESCA, P.; GALINBERT, M. Animal-Assisted Therapy in a Nursing Home Alzheimer Special Care Unit. In: INTERNACIONAL CONFERENCE ON HUMAN-ANIMAL INTERACTIONS, PEOPLE AND ANIMALS: TIMELESS RELATIONSHIP, 10, Glasglow, 2004. Pet Partners. Disponível em: <http://www.deltasociety.org> Acesso em: 29 jan. 2014.

[5] FINE, A. Handbook on Animal Assisted Therapy: Theoretical foundations and guidelines for practice. 2. ed. San Diego, CA: Academic Press, 2006.

[6] GAMMONLEY, J., \& YATES, J. Pet projects: Animal assisted therapy in nursing homes. Journal of Gerontological Nursing, 1991, v.17, n.1, p. 12-15, 1991.

[7] LERMONTOV, Tatiana. Terapia com animais. Disponível em: <http:// www.saudevidaonline.com.br/artigo 74> Acesso em: 10 fev. 2014.

[8] MARTINS, M. F. et al A Zooterapia: uma possibilidade e contribuição como dispositivo terapêutico de idosos institucionalizados. In: XXXIV CONGRESSO BRASILEIRO DE MEDICINA VETERINÁRIA, Santos, 2007. Anais. Santos: CONBRAVET, 2007.

[9] NOGUEIRA, M.J.C. Abordagem holística. In: CONGRESSO BRASILEIRO DE ENFERMAGEM, 37, Recife, 1985. Anais. Recife: Associação Brasileira de Enfermagem, 1986, p. 508-526.

[10] RAMOS, L. R. Fatores determinantes do envelhecimento saudável em idosos residentes em centro urbano. Projeto Epidoso. Rio de Janeiro, Cadernos de Saúde Pública, 2003, v.19, n.3, p. 793-798. 
[11] ZISSELMAN, M. H., ROVNER, B. W., SHMUELY, Y., \& FERRIE, P. Pet therapy intervention with geriatric psychiatry patients. The American Journal of Occupational Therapy, 1996, v.50, n.1, p. 47-50.

MARIA De fátima martins docente do Departamento de Nutrição e Produção Animal da Faculdade de Medicina Veterinária e Zootecnia da Universidade de São Paulo (FMVZ-USP), campus de Pirassununga, e coordenadora técnica do Laboratório de Ensino, Pesquisa e Extensão em Zooterapia, Helicicultura e Bem-estar Animal - e-mail:fmartins@usp.br

CARINA TORELLI bolsista do Fomento às Iniciativas de Cultura e Extensão da Pró-Reitoria de Cultura e Extensão Universitária (PRCEU-USP)

GISE LE DELA RICCI mestranda do Programa de Pós-graduação em Nutrição e Produção Animal da Faculdade de Medicina Veterinária e Zootecnia da Universidade de São Paulo (FMVZ-USP), campus de Pirassununga

thiago William De Almeida zootecnista do Programa de Treinamento Técnico da Faculdade de Medicina Veterinária e Zootecnia da Universidade de São Paulo (FMVZ-USP), campus de Pirassununga 